\title{
Combustion activities of the Ru catalysts supported on hexagonal $\mathrm{YbFeO}_{3}$
}

\author{
Yuichi MASUDA, Saburo HOSOKAWA ${ }^{\dagger}$ and Masashi INOUE
}

Department of Energy and Hydrocarbon Chemistry, Graduate School of Engineering, Kyoto University, Katsura, Kyoto $615-8510$

\begin{abstract}
Hexagonal $\mathrm{YbFeO}_{3}\left(h-\mathrm{YbFeO}_{3}\right)$ were prepared by solvothermal and Pechini methods. Although the morphology of $h$-YbFeO synthesized by solvothermal method was hexagonal plate, $h-\mathrm{YbFeO}_{3}$ synthesized by the Pechini method was composed of large aggregates of irregularly-shaped fine particles. Activities of $\mathrm{Ru}$ catalysts supported on $\boldsymbol{h}$ - $\mathrm{YbFeO}_{3}$ for combustion reaction of propane or propene were investigated. For the support of the $\mathrm{Ru}$ catalyst, $h$ - $\mathrm{YbFeO}_{3}$ synthesized by the solvothermal method was superior to that prepared by the Pechini method. Effects of the reaction temperatures of the solvothermal synthesis upon the activities of the Ru catalysts supported on the products were examined and it was found that the catalyst supported on crystallized $h-\mathrm{YbFeO}_{3}$ showed higher activity than the catalyst supported on an amorphous product having a large surface area. (C2011 The Ceramic Society of Japan. All rights reserved.
\end{abstract}

Key-words : $\mathrm{YbFeO}_{3}$, Hexagonal phase, Perovskite, Catalyst, Morphology, Combustion, XAFS, Solvothermal method

[Received June 30, 2011; Accepted August 29, 2011]

\section{Introduction}

Mixed oxides of rare earth (RE) and iron having garnet $\left(\mathrm{RE}_{3} \mathrm{Fe}_{5} \mathrm{O}_{12}\right)$, perovskite $\left(\mathrm{REFeO}_{3}\right)$ and magnetoplumbite $\left(\mathrm{RE}_{1-x} \mathrm{M}_{x} \mathrm{Fe}_{12} \mathrm{O}_{19}: \mathrm{M}=\mathrm{Ba}, \mathrm{Sr}, \mathrm{Pb}\right)$ structures have been applied in various fields such as magnetism, fuel cells (cathode material for SOFC), gas sensors, catalysts, and so on. ${ }^{1)-5)}$ Orthorhombic $\mathrm{REFeO}_{3}\left(o-\mathrm{REFeO}_{3}\right)$ with the perovskite structure has recently received much attention in the catalyst field. For example, Nishihata et al. ${ }^{6)}$ reported that $\mathrm{LaFe}_{0.57} \mathrm{Co}_{0.38} \mathrm{Pd}_{0.05} \mathrm{O}_{3}$ with the perovskite structure is successfully applied as a catalyst for the vehicle exhaust treatment. Andrews et al. reported that $\mathrm{LaFe}_{0.95} \mathrm{Pd}_{0.05} \mathrm{O}_{3}$ is an effective catalyst for Suzuki-Miyaura coupling reaction in organic chemistry. ${ }^{7)}$

$o-\mathrm{REFeO}_{3}$ with the perovskite structure $(\mathrm{Pbnm})$ is thermodynamically stable for all the RE elements, and hexagonal $\mathrm{REFeO}_{3}\left(h-\mathrm{REFeO}_{3}\right)$ belonging to the space group of $P 6_{3} \mathrm{~cm}$ is known as a metastable phase. ${ }^{8), 9)} o-\mathrm{REFeO}_{3}$ is preferentially synthesized by conventional methods such as solid state reaction or coprecipitation method: calcination at high temperatures required by these methods facilitates the formation of $o-\mathrm{REFeO}_{3}$. On the other hand, the synthesis of $h-\mathrm{REFeO}_{3}$ is rather difficult; however, several synthetic methods for $h-\mathrm{REFeO}_{3}$ have been reported; i.e., the thermal treatment of an amorphous phase obtained by hydrolysis of a yttrium-iron double alkoxide, ${ }^{10)}$ and quenching a melt $(\mathrm{RE}=\mathrm{Lu}){ }^{11)}$

We found that $h-\mathrm{REFeO}_{3}$ (Tm-Lu) was synthesized by the solvothermal reaction of $\mathrm{RE}$ acetates with iron acetylacetonate in 1,4-butanediol. ${ }^{8)}$ We also reported that $o$ - and $h$ - $\mathrm{REFeO}_{3}$ could be selectively synthesized by the choice of starting materials and the addition of suitable amines as additives. ${ }^{12)}$

Only few works have studied the catalytic property of $h-\mathrm{REFeO}_{3}$. Li et al. have studied the $\mathrm{CO}$ oxidation activity of $h-\mathrm{YFe}_{1-x} \mathrm{Pd}_{x} \mathrm{O}_{3-\delta}$ obtained by calcination of the precursor synthesized by a sol-gel process. ${ }^{13)}$ In a previous paper, we

$\uparrow$ Corresponding author: S. Hosokawa; E-mail: hosokawa@t04. mbox.media.kyoto-u.ac.jp compared the catalytic activities of $o$ - and $h-\mathrm{YbFeO}_{3}$ phases and found that the $h-\mathrm{YbFeO}_{3}$ sample, which had a high surface area $\left(29 \mathrm{~m}^{2} \mathrm{~g}^{-1}\right)$ even after calcination at $800^{\circ} \mathrm{C}$, had a higher catalytic activity for methane combustion than the $o-\mathrm{YbFeO}_{3}$ sample calcined at $800^{\circ} \mathrm{C}^{14)}$

In this work, properties of $h$ - and $o-\mathrm{YbFeO}_{3}$ samples for the supports of the noble metal catalysts were examined: combustion reactions of propane or propene on these catalysts were investigated. Since we found that the $h-\mathrm{YbFeO}_{3}$ could be synthesized by the Pechini method, the effect of preparation method on catalytic activity was also examined.

\section{Experimental}

$h-\mathrm{YbFeO}_{3}$ was prepared by the solvothermal (ST) method. Ytterbium acetate hydrate $\left[\mathrm{Yb}(\mathrm{OAc})_{3} \cdot 4 \mathrm{H}_{2} \mathrm{O}\right.$, Wako], and iron acetylacetonate $\left[\mathrm{Fe}(\mathrm{acac})_{3}\right.$, Dojin] were used without further purification. $\mathrm{Yb}(\mathrm{OAc})_{3}(15 \mathrm{mmol})$ and $\mathrm{Fe}(\mathrm{acac})_{3} \quad(15 \mathrm{mmol})$ were suspended with the aid of ultrasonication in $120 \mathrm{ml}$ of 1,4-butanediol $(1,4-\mathrm{BG})$ in a test tube serving as autoclave liner. The test tube was placed in a $300 \mathrm{~mL}$ autoclave. An additional $30 \mathrm{~mL}$ of 1,4-BG was placed in the gap between the autoclave wall and the test tube. The autoclave was purged with nitrogen, heated to $230,250,285$ or $315^{\circ} \mathrm{C}$ at a rate of $2.3^{\circ} \mathrm{C} \mathrm{min}^{-1}$ and kept at that temperature for $2 \mathrm{~h}$. After the assembly was cooled to room temperature, the resulting product was centrifuged. To the sedimented part of the product, methanol was added and vigorously mixed to peptize the colloidal particles. The mixture was centrifuged saving the supernatant. This procedure was repeated for several times, finally discarding the sediment. All the supernatant parts ware gathered together, and a small amount of the concentrated ammonia solution was added to coagulate the particles. The product was repeatedly washed with methanol and dried in air. The ceramic yield of the product was above $80 \%$. These samples are designated as $h-\mathrm{YbFeO}_{3}(\mathrm{ST}-\mathrm{xx})$, in which $\mathrm{xx}$ is the temperature for the solvothermal reaction.

For comparison, $h-\mathrm{YbFeO}_{3}$ was also synthesized by the Pechini (PC) method. $0.4 \mathrm{~mol}$ of citric acid was dissolved in $180 \mathrm{ml}$ of water at $80^{\circ} \mathrm{C}$. Then, iron nitrate nonahydrate 
$\left[\mathrm{Fe}\left(\mathrm{NO}_{3}\right)_{3} \cdot 9 \mathrm{H}_{2} \mathrm{O}, 10 \mathrm{mmol}\right]$ and ytterbium carbonate hydrate $\left[\mathrm{Yb}_{2}\left(\mathrm{CO}_{3}\right)_{3} \cdot n \mathrm{H}_{2} \mathrm{O}, 5 \mathrm{mmol}\right]$ were added and the mixture was magnetically stirred for $2 \mathrm{~h}$ at $80^{\circ} \mathrm{C}$ to obtain a solution containing metal citrate complexes. Ethylene glycol $(0.4 \mathrm{~mol})$ was added to this solution and the solution was stirred at $130^{\circ} \mathrm{C}$ for $3 \mathrm{~h}$ to obtain a gelatinous solution. After the gel was heated in a mantle heater at $350^{\circ} \mathrm{C}$ for over $5 \mathrm{~h}$, the thus-obtained brown powder was calcined at 700 or $800^{\circ} \mathrm{C}$ for $2 \mathrm{~h}$. These samples were designated as $h-\mathrm{YbFeO}_{3}(\mathrm{PC}-\mathrm{yy})$, in which yy is the calcination temperature.

Supported catalysts were prepared by an impregnation method: $1.0 \mathrm{~g}$ of a support was added to a solution of $\mathrm{Ru}(\mathrm{acac})_{3}, \mathrm{Rh}(\mathrm{acac})_{3}$ or $\mathrm{Pd}(\mathrm{OAc})_{2}$ in $10 \mathrm{ml}$ of THF or methanol at room temperature, and after the evaporation of the solvent, the resulting powder was calcined at $500^{\circ} \mathrm{C}$ for $30 \mathrm{~min}$ to afford the noble-metal/support catalysts. The molar ratio of noble-metal/Fe was fixed to 0.05 . $\mathrm{A} \mathrm{Ru} / \mathrm{Al}_{2} \mathrm{O}_{3}$ catalyst was prepared by the same impregnation method. Commercial $\mathrm{Al}_{2} \mathrm{O}_{3}$ (Sumitomo Chemical Co., Ltd., AKP-G015; JRC-ALO-8 equivalent) was used without further purification. The Ru loading was $2 \mathrm{wt} \%$ on a metal basis.

Catalyst tests for propane or propene combustion were performed in a fixed-bed reactor. The catalyst was tabletted, pulverized into 10-22 mesh, and set in the reactor. The reaction gas composed of $3000 \mathrm{ppm}$ hydrocarbons, 17 or $14 \% \mathrm{O}_{2}$, and $\mathrm{He}$ balance was introduced to the catalyst bed at $W / F=0.3 \mathrm{~g} \mathrm{~s} \mathrm{ml}^{-1}$. The effluent gases from the reactor were analyzed with a gas chromatograph (GC-8A, Porapak Q).

The physical properties of the products were analyzed by X-ray powder diffraction (XRD: Shimadzu XD-D1), by the BET single point method (Micromeritics Flowsorb II 2300 sorptionmeter), and by a transmission electron microscope (TEM: Hitachi $\mathrm{H}-800$ ). Ru K-edge X-ray absorption fine structure (XAFS) measurements were performed at the BL01B1 beamline of SPring- 8 with the approval of the Japan Synchrotron Radiation Research Institute (JASRI). The storage ring was operated at $8 \mathrm{GeV}$ with a ring current of $98-100 \mathrm{~mA}$. A double-mirror system was used to avoid higher harmonics in the X-ray beam. A Si(311) two crystal monochromator was used. Ru K-edge XAFS spectra were measured in a transmission mode using $\mathrm{I}_{0}[100 \% \mathrm{Ar}]$ and $\mathrm{I}$ [75\% Ar diluted with $\mathrm{Kr}$ ] ion chambers or in a fluorescence mode using an $\mathrm{I}_{0}$ ion chamber $[100 \% \mathrm{Ar}]$ and a Lytle detector $[100 \% \mathrm{Kr}]$. X-ray absorption near-edge structure (XANES) and extended X-ray absorption fine structure (EXAFS) data were analyzed using the REX2000 program (ver. 2.5; Rigaku Corp.).

\section{Results and discussion}

\subsection{Syntheses of $h-\mathrm{YbFeO}_{3}$ by solvothermal and Pechini methods}

Figure 1 shows the XRD patterns of the products assynthesized by solvothermal reactions at various temperatures. The diffraction peaks due to $h-\mathrm{YbFeO}_{3}$ were clearly observed for the products as-synthesized at above $250^{\circ} \mathrm{C}$. On the other hand, the product obtained by the solvothermal reaction at $230^{\circ} \mathrm{C}$ exhibited the diffraction peaks with significantly low intensities, indicating that this product was essentially amorphous.

With the increase in the solvothermal temperature, the BET surface area of the $h-\mathrm{YbFeO}_{3}$ sample decreased, while the crystallite size as well as the aspect ratio $\left(D_{300} / D_{002}\right)$ of the sample increased (Table 1). The last result indicates that the crystal growth along the $a b$ plane proceeded preferentially over the growth toward the $c$ axis direction, the preferential growth becoming more significant with the increase in the solvothermal temperature. The TEM observation (Fig. 2) revealed that the

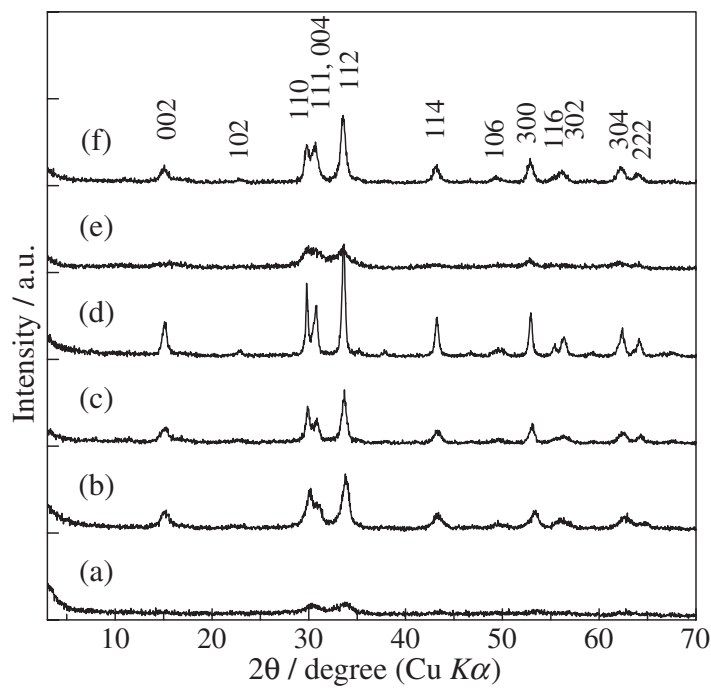

Fig. 1. XRD patterns of the products as-synthesized by the solvothermal and Pechini methods: (a), $\mathrm{YbFeO}_{3}(\mathrm{ST}-230)$; (b), $h$ - $\mathrm{YbFeO}_{3}(\mathrm{ST}-250)$; (c), $h$-YbFeO ${ }_{3}(\mathrm{ST}-285)$; (d), $h-\mathrm{YbFeO}_{3}(\mathrm{ST}-315)$; (e), $\mathrm{YbFeO}_{3}(\mathrm{PC}-700)$; (f), $h-\mathrm{YbFeO}_{3}(\mathrm{PC}-800)$.

Table 1. BET surface areas and crystallite sizes of $h-\mathrm{YbFeO}_{3}$ samples as-synthesized by the solvotheramal method at various temperatures and by Pechini method

\begin{tabular}{|c|c|c|c|c|c|c|}
\hline \multirow[t]{2}{*}{ Entry } & \multirow[t]{2}{*}{ Sample } & \multicolumn{2}{|c|}{$\begin{array}{c}\text { Crystallite } \\
\text { size/nm }\end{array}$} & \multirow[t]{2}{*}{$D_{300} / D_{002}$} & \multirow{2}{*}{$\begin{array}{c}S_{\mathrm{BET}} \\
/ \mathrm{m}^{2} \mathrm{~g}^{-1}\end{array}$} & \multirow{2}{*}{$\begin{array}{c}S_{\mathrm{G}} \\
/ \mathrm{m}^{2} \mathrm{~g}^{-1}\end{array}$} \\
\hline & & $D_{300}$ & $D_{002}$ & & & \\
\hline 1 & $\mathrm{YbFeO}_{3}(\mathrm{ST}-230)$ & - & - & - & 155 & - \\
\hline 2 & $h-\mathrm{YbFeO}_{3}(\mathrm{ST}-250)$ & 8 & 7 & 1.1 & 72 & $86^{\mathrm{a})}$ \\
\hline 3 & $h-\mathrm{YbFeO}_{3}(\mathrm{ST}-285)$ & 25 & 10 & 2.5 & 54 & $52^{\mathrm{b})}$ \\
\hline 4 & $h-\mathrm{YbFeO}_{3}(\mathrm{ST}-315)$ & 58 & 18 & 3.2 & 26 & $26^{\mathrm{b})}$ \\
\hline 5 & $h-\mathrm{YbFeO}_{3}(\mathrm{PC}-800)$ & 19 & 12 & 1.6 & 30 & $55^{\mathrm{b})}$ \\
\hline
\end{tabular}

a) Geometric surface area calculated on the assumption that each $h$ - $\mathrm{YbFeO}_{3}$ particle is spherical with a diameter of $7 \mathrm{~nm}$.

b) Geometric surface area calculated on the assumption that each $h$ - $\mathrm{YbFeO}_{3}$ particle is a hexagonal plate with a thickness of $D_{002}$ and a width of $D_{300}$.

morphology of $h$-YbFeO $3(\mathrm{ST}-315)$ was hexagonal plate, as reported in previous works, ${ }^{13), 14)}$ while $h-\mathrm{YbFeO}_{3}(\mathrm{ST}-250)$ was composed of nanoparticles with the sizes of $10 \mathrm{~nm}$. These results agreed well with the aspect ratio determined by XRD analyses.

As for the Pechini method, the product obtained by calcination at $700^{\circ} \mathrm{C}$ was essentially amorphous, while $h-\mathrm{YbFeO}_{3}$ was crystallized by calcination at $800^{\circ} \mathrm{C}$. Although the crystallite size of $h-\mathrm{YbFeO}_{3}(\mathrm{PC}-800)$ was smaller than that of $h-\mathrm{YbFeO}_{3}(\mathrm{ST}-315)$, the BET surface area of the former sample was smaller than that of the latter, indicating that the particles of $h-\mathrm{YbFeO}_{3}(\mathrm{PC}-800)$ were severely aggregated. Actually, the TEM images of $h-\mathrm{YbFeO}_{3}(\mathrm{PC}-800)$ showed that this product was composed of large aggregates of irregularly-shaped particles. The aspect ratio of $h-\mathrm{YbFeO}_{3}(\mathrm{PC}-800)$ was much smaller than that of $h-\mathrm{YbFeO}_{3}(\mathrm{ST}-315)$.

\subsection{Combustion activities of noble metal catalysts supported on $h-\mathrm{YbFeO}_{3}$}

Figure 3 shows the XRD patterns of the Ru catalysts obtained by calcination at $500^{\circ} \mathrm{C}$ after impregnation of $\mathrm{Ru}(\mathrm{acac})_{3}$. The peaks due to $\mathrm{Ru}$ species were not observed, and the patterns were essentially identical with those of the as-synthesized $h-\mathrm{YbFeO}_{3}$ 

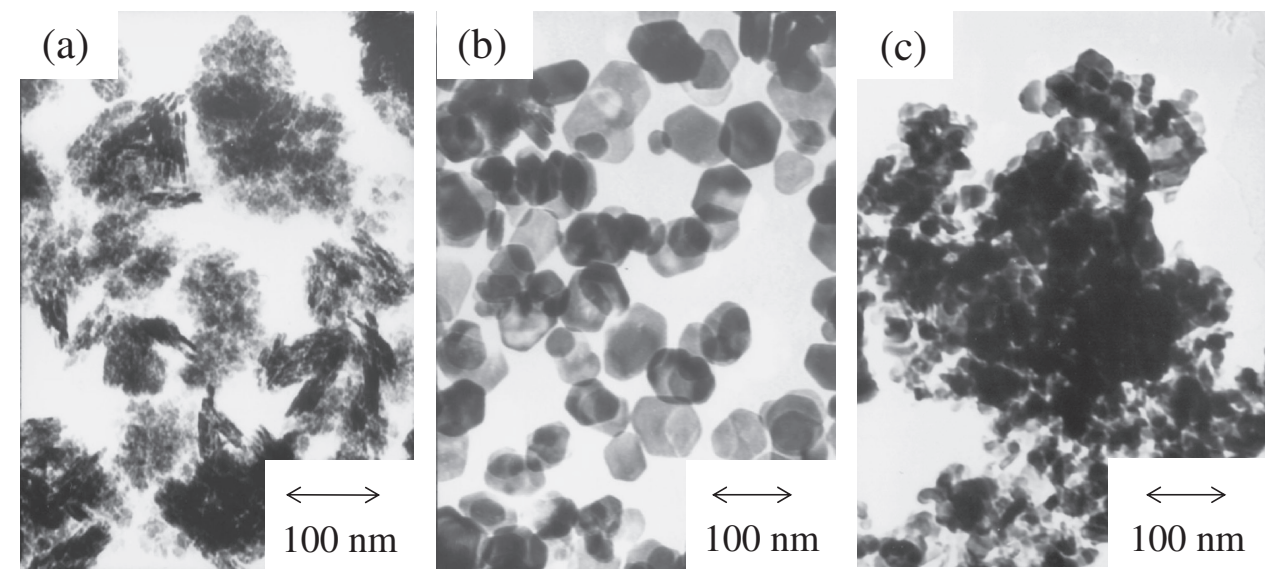

Fig. 2. TEM images of the products as-synthesized by the solvothermal and Pechini methods: (a), $h$ - $\mathrm{YbFeO}_{3}(\mathrm{ST}-250$ ); (b), $h$ - $\mathrm{YbFeO}_{3}$ (ST-315); (c), $h$ - $\mathrm{YbFeO}_{3}(\mathrm{PC}-800)$

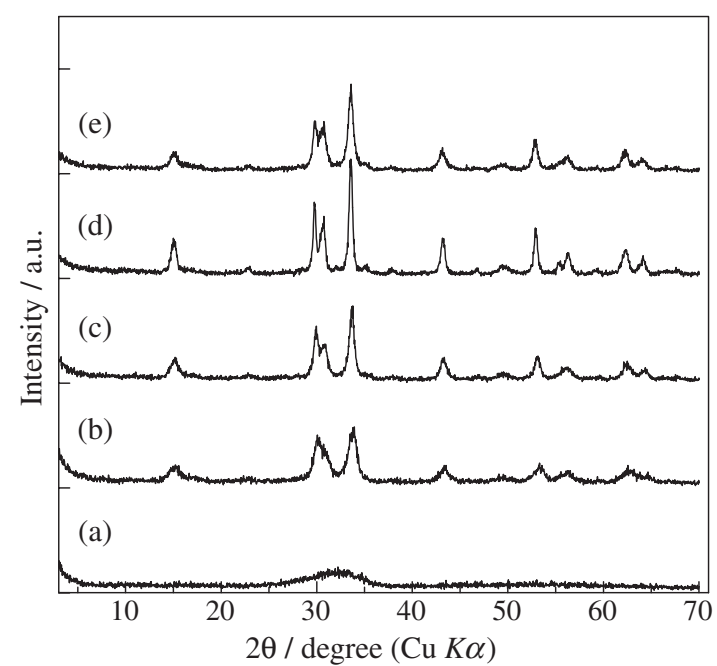

Fig. 3. XRD patterns of Ru catalysts obtained by calcination at $500^{\circ} \mathrm{C}$ after impregnation method of $\mathrm{Ru}(\mathrm{acac})_{3}:$ (a), $\mathrm{Ru} / \mathrm{YbFeO}_{3}(\mathrm{ST}-230)$; (b), $\mathrm{Ru} / h-\mathrm{YbFeO}_{3}(\mathrm{ST}-250) ; \quad(\mathrm{c}), \quad \mathrm{Ru} / h-\mathrm{YbFeO}_{3}(\mathrm{ST}-285) ; \quad(\mathrm{d}), \quad \mathrm{Ru} /$ $h$ - $\mathrm{YbFeO}_{3}(\mathrm{ST}-315)$; (e), $\mathrm{Ru} / h-\mathrm{YbFeO}_{3}(\mathrm{PC}-800)$. samples shown in Fig. 1. Although the BET surface area of $\mathrm{Ru} / \mathrm{YbFeO}_{3}(\mathrm{ST}-230)$ drastically decreased by calcination at $500^{\circ} \mathrm{C}$ after the impregnation of $\mathrm{Ru}(\mathrm{acac})_{3}$, both the BET surface areas of the $\mathrm{Ru}$ catalysts and crystallite sizes of the $h-\mathrm{YbFeO}_{3}$ phase (Table 2) were essentially identical with those of the corresponding as-synthesized $h-\mathrm{YbFeO}_{3}$ samples (Table 1), indicating that the sintering of $h-\mathrm{YbFeO}_{3}$ scarcely occurred by calcination at $500^{\circ} \mathrm{C}$. This result is consistent with the previously reported result that the hexagonal-plate morphology of $h-\mathrm{YbFeO}_{3}$ synthesized by the solvothermal method was maintained during calcination until the phase transformation into the thermodynamically stable $o-\mathrm{YbFeO}_{3}$ occurred at $\sim 950^{\circ} \mathrm{C}^{8), 14)}$

Table 2 shows that the activities of the $h-\mathrm{YbFeO}_{3}$-supported noble metal catalysts for combustion reactions. Of the noble metal catalysts supported on $h-\mathrm{YbFeO}_{3}(\mathrm{ST}-315)$, the Ru catalyst showed the highest activity for propane combustion, while the Pd catalyst had a higher activity than the $\mathrm{Ru}$ catalyst for propene combustion. The $\mathrm{Ru} / h-\mathrm{YbFeO}_{3}(\mathrm{ST}-315)$ had a higher activity than $\mathrm{Ru} / o-\mathrm{YbFeO}_{3}$ or $\mathrm{Ru} / h-\mathrm{YbFeO}_{3}(\mathrm{PC}-800)$ for both the propane and propene combustions, suggesting that the catalytic activity was affected on the crystal structure and/or morphology of the support particles. The catalytic activity of

Table 2. Combustion activities, BET surface areas and crystallite sizes of noble metal catalysts obtained by calcination at $500^{\circ} \mathrm{C}$ after impregnation method

\begin{tabular}{|c|c|c|c|c|c|c|c|c|}
\hline \multirow{2}{*}{ Entry } & \multirow{2}{*}{ Catalyst } & \multicolumn{2}{|c|}{$\mathrm{C}_{3} \mathrm{H}_{8}{ }^{\mathrm{a})}$} & \multicolumn{2}{|c|}{$\mathrm{C}_{3} \mathrm{H}_{6}{ }^{\mathrm{b})}$} & \multirow{2}{*}{$S_{\mathrm{BET}} / \mathrm{m}^{2} \mathrm{~g}^{-1}$} & \multicolumn{2}{|c|}{ Crystallite size $/ \mathrm{nm}$} \\
\hline & & $T_{50}{ }^{\mathrm{c}} /{ }^{\circ} \mathrm{C}$ & $T_{90}{ }^{\mathrm{d})} /{ }^{\circ} \mathrm{C}$ & $T_{50}{ }^{\mathrm{c}} /{ }^{\circ} \mathrm{C}$ & $T_{90}{ }^{\mathrm{d})} /{ }^{\circ} \mathrm{C}$ & & $D_{300}$ & $D_{002}$ \\
\hline 1 & $\left.h-\mathrm{YbFeO}_{3}(\mathrm{ST}-315)^{\mathrm{e}}\right)$ & 407 & 473 & 366 & 423 & 26 & 47 & 18 \\
\hline 2 & $\mathrm{Pd} / h-\mathrm{YbFeO}_{3}(\mathrm{ST}-315)$ & 284 & 322 & 187 & 197 & - & - & - \\
\hline 3 & $\mathrm{Rh} / h-\mathrm{YbFeO}_{3}(\mathrm{ST}-315)$ & 232 & 262 & 242 & 268 & - & - & - \\
\hline 4 & $\mathrm{Ru} / h-\mathrm{YbFeO}_{3}(\mathrm{ST}-315)$ & 214 & 256 & 201 & 220 & 32 & 40 & 15 \\
\hline 5 & $\mathrm{Ru} / o-\mathrm{YbFeO}_{3}{ }^{\mathrm{f}}{ }^{\mathrm{f}}$ & 267 & 298 & 233 & 264 & 20 & - & - \\
\hline 6 & $\mathrm{Ru} / \mathrm{Al}_{2} \mathrm{O}_{3}$ & 246 & 285 & 234 & 247 & 148 & - & - \\
\hline 7 & $\mathrm{Ru} / h-\mathrm{YbFeO}_{3}(\mathrm{PC}-800)$ & 239 & 286 & 207 & 223 & 36 & 22 & 6 \\
\hline 8 & $\mathrm{Ru} / \mathrm{YbFeO}_{3}(\mathrm{ST}-230)$ & 247 & 314 & 192 & 218 & 93 & - & - \\
\hline 9 & $\mathrm{Ru} / h-\mathrm{YbFeO}_{3}(\mathrm{ST}-250)$ & 210 & 254 & 176 & 195 & 72 & 11 & 6 \\
\hline 10 & $\mathrm{Ru} / h-\mathrm{YbFeO}_{3}(\mathrm{ST}-285)$ & 201 & 241 & 185 & 217 & 47 & 18 & 10 \\
\hline
\end{tabular}

a) Reaction conditions: $\mathrm{C}_{3} \mathrm{H}_{8}, 0.3 \% ; \mathrm{O}_{2}, 17 \%$, He balance; $W / F=0.3 \mathrm{~g} \mathrm{~s} \mathrm{ml}^{-1}$.

b) Reaction conditions: $\mathrm{C}_{3} \mathrm{H}_{6}, 0.3 \% ; \mathrm{O}_{2}, 14 \%$, He balance; $W / F=0.3 \mathrm{~g} \mathrm{~s} \mathrm{ml}^{-1}$.

c) Temperature at which the catalyst attained $50 \%$ conversion of hydrocarbons.

d) Temperature at which the catalyst attained $90 \%$ conversion of hydrocarbons.

e) The sample was calcined at $500^{\circ} \mathrm{C}$.

f) $o-\mathrm{YbFeO}_{3}$ was synthesized by a solvothermal method. ${ }^{12)}$ 
$\mathrm{Ru} / h-\mathrm{YbFeO}_{3}(\mathrm{ST}-315)$ was superior to that of $\mathrm{Ru} / \mathrm{Al}_{2} \mathrm{O}_{3}$, even though the latter catalyst had a large surface area.

Entries $8-10$ of Table 2 examined the effect of the temperature of solvothermal reaction for the synthesis of $h-\mathrm{YbFeO}_{3}$ upon the catalytic activity. $\mathrm{Ru} / h-\mathrm{YbFeO}_{3}(\mathrm{ST}-250)$ and $\mathrm{Ru} /$ $h$ - $\mathrm{YbFeO}_{3}(\mathrm{ST}-285)$ had essentially identical activities with that of $\mathrm{Ru} / h-\mathrm{YbFeO}_{3}(\mathrm{ST}-315)$ for the propane combustion reaction, while $\mathrm{Ru} / \mathrm{YbFeO}_{3}(\mathrm{ST}-230)$ had a much lower catalytic activity. In propene combustion reaction, the catalytic activity of $\mathrm{Ru} /$ $h-\mathrm{YbFeO}_{3}$ slightly increased with the decrease in solvothermal temperature from $315^{\circ} \mathrm{C}$ to $250^{\circ} \mathrm{C}$, while $\mathrm{Ru} / h-\mathrm{YbFeO}_{3}(\mathrm{ST}-230)$ showed a low activity. These results suggest the crystallized $h-\mathrm{YbFeO}_{3}$ was effective as catalyst support for the combustion reaction.

The states of $\mathrm{Ru}$ species on the supports were investigated by $\mathrm{Ru}$ K-edge XAFS analyses. As shown in Fig. 4, the Ru

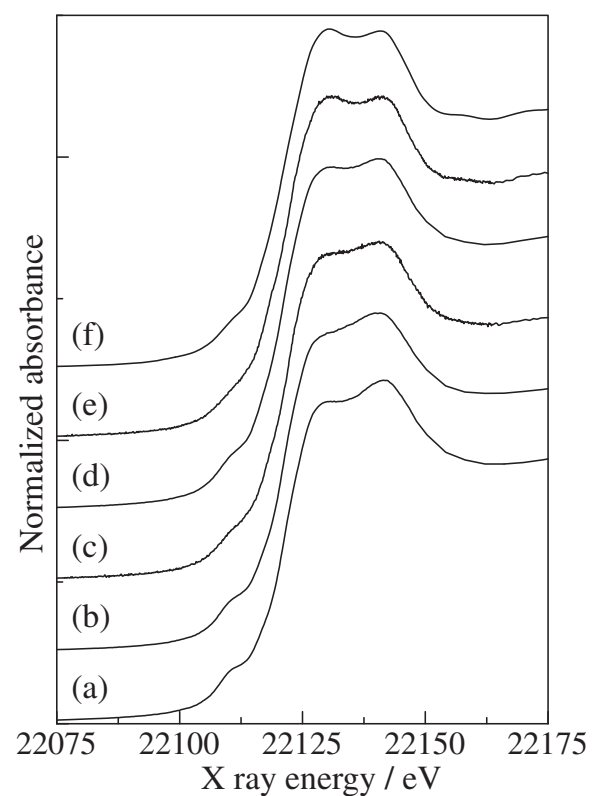

Fig. 4. XANES spectra of: (a), $\mathrm{Ru} / \mathrm{YbFeO}_{3}(\mathrm{ST}-230) ; \quad$ (b), $\mathrm{Ru} /$ $h-\mathrm{YbFeO}_{3}(\mathrm{ST}-250) ; \quad$ (c), $\mathrm{Ru} / h-\mathrm{YbFeO}_{3}(\mathrm{ST}-285) ; \quad$ (d), $\mathrm{Ru} /$ $h-\mathrm{YbFeO}_{3}(\mathrm{ST}-315)$; (e), $\mathrm{Ru} / h-\mathrm{YbFeO}_{3}(\mathrm{PC}-800)$; (f), $\mathrm{RuO}_{2}$.

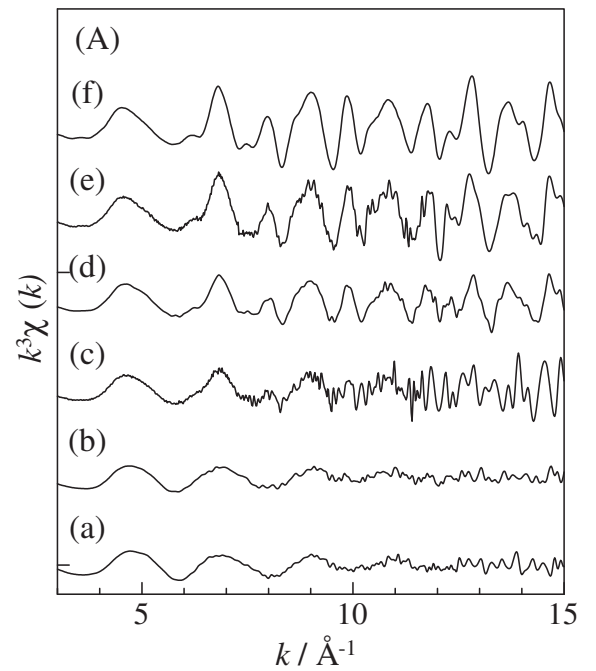

K-edge XANES spectra of $\mathrm{Ru} / h-\mathrm{YbFeO}_{3}(\mathrm{ST}-315)$ and $\mathrm{Ru} /$ $h-\mathrm{YbFeO}_{3}(\mathrm{PC}-800)$ catalysts were essentially identical with that of the $\mathrm{RuO}_{2}$ standard, indicating that the $\mathrm{Ru}$ species in these catalysts have a $\mathrm{RuO}_{2}$-like structure. The XANES spectra of $\mathrm{Ru} / h-\mathrm{YbFeO}_{3}(\mathrm{ST}-250)$ and $\mathrm{Ru} / \mathrm{YbFeO}_{3}(\mathrm{ST}-230)$ were different from that of the $\mathrm{RuO}_{2}$ standard in that the intensities of the peak at around $22125 \mathrm{eV}$ of the former samples were weaker than that of the $\mathrm{RuO}_{2}$ standard. Moreover, $\mathrm{Ru} / h-\mathrm{YbFeO}_{3}(\mathrm{ST}-250)$ and $\mathrm{Ru} / h-\mathrm{YbFeO}_{3}(\mathrm{ST}-230)$ exhibited a pre-edge peak at around $22110 \mathrm{eV}$. The existence of the pre-edge peak implies that the $\mathrm{Ru}$ species is distorted from the octahedral symmetry, ${ }^{15), 16)}$ suggesting that the distorted $\mathrm{Ru}$ species were formed on $h$ - $\mathrm{YbFeO}_{3}(\mathrm{ST}-250)$ and $\mathrm{YbFeO}_{3}(\mathrm{ST}-230)$.

In the Fourier transforms (FT) of Ru K-edge EXAFS spectra (Fig. 5), the peaks at 2.0-4.0 $\AA$ were observed for $\mathrm{Ru} /$ $h$ - $\mathrm{YbFeO}_{3}(\mathrm{ST}-315)$ and $\mathrm{Ru} / h-\mathrm{YbFeO}_{3}(\mathrm{PC}-800)$, and the oscillation and the feature of the FT spectra were essentially identical with those of the $\mathrm{RuO}_{2}$ standard, indicating that the major part of $\mathrm{Ru}$ species was loaded as $\mathrm{RuO}_{2}$. On the other hand, the intensity of the peaks due to the second shell of the Ru species of the $\mathrm{Ru} / h-\mathrm{YbFeO}_{3}$ catalyst decreased with the decrease in the solvothermal temperature for the synthesis of the $h-\mathrm{YbFeO}_{3}$ phase, suggesting that the particle size of the $\mathrm{RuO}_{2}$-like phase decreased with the increase in the surface area of the support. The second shell for $\mathrm{Ru} / h-\mathrm{YbFeO}_{3}(\mathrm{ST}-250)$ or $\mathrm{Ru} / \mathrm{YbFeO}_{3}(\mathrm{ST}-230)$ was not observed in the FT of the EXAFS spectra, indicating that the $\mathrm{Ru}$ species were highly dispersed on the surface of $h$ - $\mathrm{YbFeO}_{3}(\mathrm{ST}-250)$ or $\mathrm{YbFeO}_{3}(\mathrm{ST}-230)$ because of strong interaction with the support.

Although the dispersion state of $\mathrm{Ru}$ species on $h$ - $\mathrm{YbFeO}_{3}$ (ST-250) was significantly different from that on $h-\mathrm{YbFeO}_{3}(\mathrm{ST}-315)$, the catalytic activity of $\mathrm{Ru} / \mathrm{YbFeO}_{3}(\mathrm{ST}-250)$ for propane combustion was essentially identical with that of $\mathrm{Ru} / h-\mathrm{YbFeO}_{3}(\mathrm{ST}-315)$. On the other hand, the catalytic activity for propene combustion increased with the increase in the dispersion of $\mathrm{Ru}$ species when they were supported on crystallized $h-\mathrm{YbFeO}_{3}$. This result suggests that the nature of $\mathrm{Ru}$ species affected the catalytic activity in combustion of propene possessing both allylic hydrogen atoms having high reactivity and an unsaturated $\mathrm{C}=\mathrm{C}$ bond having high coordination ability to the catalyst.

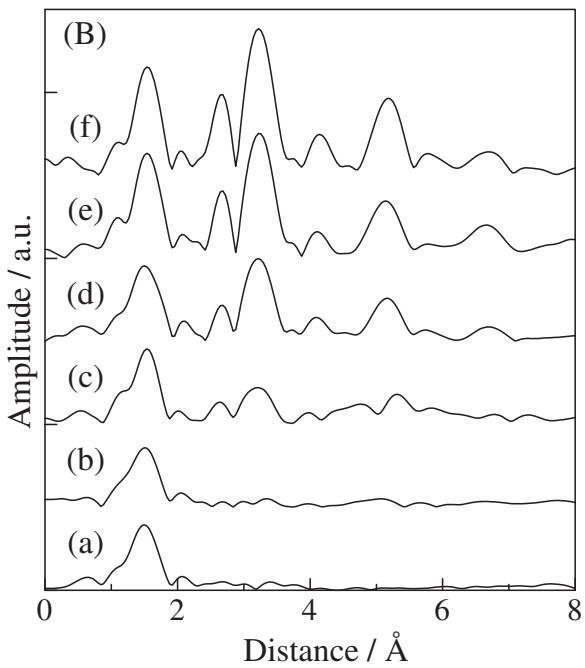

Fig. 5. Ru K-edge EXAFS (A) and FT (B) spectra of: (a), Ru/YbFeO $3(\mathrm{ST}-230)$; (b), Ru/h-YbFeO 3 (ST-250); (c), Ru/ $h-\mathrm{YbFeO}_{3}(\mathrm{ST}-285) ;(\mathrm{d}), \mathrm{Ru} / h-\mathrm{YbFeO}_{3}(\mathrm{ST}-315) ;(\mathrm{e}), \mathrm{Ru} / h-\mathrm{YbFeO}_{3}(\mathrm{PC}-800)$; (f), $\mathrm{RuO}_{2}$. 


\section{Conclusions}

Crystallized $h-\mathrm{YbFeO}_{3}$ was directly obtained by solvothermal reaction of ytterbium acetate and iron acetylacetonate at above $250^{\circ} \mathrm{C}$, while the product obtained by the reaction at $230^{\circ} \mathrm{C}$ was amorphous. $h-\mathrm{YbFeO}_{3}$ synthesized by solvothermal reaction was composed of hexagonal-plate particles. On the other hand, irregularly-shaped particles were obtained by the Pechini method.

Of the noble metal catalysts supported on $h-\mathrm{YbFeO}_{3}$, the $\mathrm{Ru}$ catalyst showed the highest activity for propane combustion reaction, and $\mathrm{Ru} / h-\mathrm{YbFeO}_{3}(\mathrm{ST}-315)$ had a higher activity than $\mathrm{Ru} / h-\mathrm{YbFeO}_{3}(\mathrm{PC}-800), \mathrm{Ru} / o-\mathrm{YbFeO}_{3}$ or $\mathrm{Ru} / \mathrm{Al}_{2} \mathrm{O}_{3}$ for both the propane and propene combustion reactions. The $\mathrm{Ru}$ catalyst supported on crystallized $h$ - $\mathrm{YbFeO}_{3}(\mathrm{ST}-250)$ showed higher activity than the Ru catalyst supported on an amorphous product $\left[\mathrm{YbFeO}_{3}(\mathrm{ST}-230)\right]$ having a large surface area. The dispersion of $\mathrm{Ru}$ species on $h-\mathrm{YbFeO}_{3}$ increased with the increase in the surface areas of the support which, in turn, increased with the decrease in the solvothermal temperature for the synthesis of $h-\mathrm{YbFeO}_{3}$. Although the dispersion of $\mathrm{Ru}$ species affected the catalytic activity for propene combustion, the catalytic activity for propane combustion did not depend on the dispersion of $\mathrm{Ru}$ species.

Acknowledgements The XAFS experiments have been performed with the approval of SPring-8 (Proposal No. 2009B1356). The authors thank Dr. Seiji Yamazoe of Ryukoku University for the measurements of XAFS spectra. This research was supported by a Grant-in-Aid for Scientific Research (No. 23685046) from the Ministry of Education, Culture, Sports, Science and Technology, Japan.

\section{References}

1) N. Q. Minh, J. Am. Ceram. Soc., 76, 563-588 (1993).

2) M. C. Carotta, G. Martinelli, Y. Sadaoka, P. Nunziante and E. Traversa, Sens. Actuators, B, 48, 270-276 (1998).

3) S. Royer, A. Van Neste, R. Davidson, S. McIntyre and S. Kaliaguine, Ind. Eng. Chem. Res., 43, 5670-5680 (2004).

4) S. Royer, F. Bērubē and S. Kaliaguine, Appl. Catal., A, 282, 273-284 (2005).

5) Z. Hába and D. Brož, J. Magn. Magn. Mater., 124, 27-30 (1993).

6) Y. Nishihata, J. Mizuki, T. Akao, H. Tanaka, M. Uenishi, M. Kimura, T. Okamoto and N. Hamada, Nature, 418, 164-167 (2002).

7) S. P. Andrews, A. F. Stepan, H. Tanaka, S. V. Ley and M. D. Smith, Adv. Synth. Catal., 347, 647-654 (2005).

8) M. Inoue, T. Nishikawa, T. Nakamura and T. Inui, J. Am. Ceram. Soc., 80, 2157-2160 (1997).

9) E. Magome, C. Moriyoshi, Y. Kuroiwa, A. Masuno and H. Inoue, Jpn. J. Appl. Phys., 49, 09ME06 (2010).

10) O. Yamaguchi, H. Takemura, M. Yamashita and A. Hayashida, J. Electrochem. Soc., 138, 1492-1494 (1991).

11) M. S. V. Kumar, K. Nagashio, T. Hibiya and K. Kuribayashi, J. Am. Ceram. Soc., 91, 806-812 (2008).

12) S. Hosokawa, H.-J. Jeon, S. Iwamoto and M. Inoue, J. Am. Ceram. Soc., 92, 2847-2853 (2009).

13) J. Li, U. G. Singh, T. D. Schladt, J. K. Stalick, S. L. Scott and R. Seshadri, Chem. Mater, 20, 6567-6576 (2008).

14) S. Hosokawa, H.-J. Jeon and M. Inoue, Res. Chem. Intermed, 37, 291-296 (2011).

15) S. Hosokawa, S. Nogawa, M. Taniguchi, K. Utani, H. Kanai and S. Imamura, Appl. Catal., A, 288, 67-73 (2005).

16) K. Getty, M. U. Delgado-Jaime and P. Kennepohl, Inorg. Chim. Acta, 361, 1059-1065 (2008). 